\title{
Revenue in U.S. hospital based outpatient wound centers: I mplications for creating accountable care organizations
}

\author{
Caroline E. Fife ${ }^{1}$, Vonda Wall ${ }^{1}$, Marissa J. Carter ${ }^{2}$, David Walker ${ }^{1}$, Brett Thomson ${ }^{1}$, Toni Turner ${ }^{3}$ \\ 1. Intellicure, Inc., The Woodlands, TX, USA. 2. Strategic Solutions, Inc., Cody, WY, USA. 3. InRich Advisors, The \\ Woodlands, TX, USA
}

Correspondence: Caroline E. Fife. Address: Intellicure, Inc., 2700 Technology Forest Blvd, Suite 250, The Woodlands, TX, 77381, USA. E-mail: cfife@intellicure.com

Received: September 2, 2012

Accepted: February 15, 2013

Online Published: February 22, 2013

DOI : $10.5430 /$ jha.v2n3p38

URL: http://dx.doi.org/10.5430/jha.v2n3p38

\section{Abstract}

Background: One of the experimental health care payment and delivery programs proposed under the auspices of the U.S. Center for Medicare and Medicaid Innovation is the Accountable Care Organization (ACO). An example of this is the physician hospital organization (PHO), an association of one or more hospitals and a group of physicians which would "bundle" their payments for an episode of care. However, as potential PHO members consider such joint ventures, they require real-world revenue data from which to determine the relative contribution of the physician and the facility to the collective bill in order to make decisions about the contract structure. In the past, physicians and hospitals have not shared cost and charge data with each other.

Methods: Using sophisticated electronic medical record software, real-world billing data were obtained from 3 hospital-based outpatient wound centers and the physicians practicing in them (1 full time equivalent each) in order to establish the relative contribution of each entity to a potential PHO.

Results: A total of 6,762 patient visits occurred, comprising 887 initial consultations and 5,875 follow-up visits. Based upon Medicare-allowable reimbursement rates, mean physician revenues represented approximately one quarter of total revenue while procedures provided almost three quarters of the global revenue, on average.

Conclusions: Among hospital-based wound centers our results confirm that procedures represent the majority of revenue for both the facility and the physician. Our results represent a starting point for hospitals and physicians to negotiate bundled payments as they attempt to transition to a value- and quality-based model of care. A sophisticated EHR designed specifically for the purpose of capturing charge data, provides the mechanism for future cost effectiveness studies.

\section{Key words}

EM coding, Center for Medicare Services, Medicare, Wound care, Level of service, Electronic medical records

\section{Background}

The impending bankruptcy of the U.S. Medicare Trust Fund has created an urgent need to deliver more cost-effective care to all Americans, particularly those with chronic illnesses, such as diabetic foot ulcers and other refractory wounds, which 
result in long-term and recurring treatment courses. In 2005, the Centers for Medicare and Medicaid Services (CMS) estimated that at least $2 \%$ of the total U.S. population suffered from a chronic wound (15\% of the elderly) ${ }^{[1]}$. In the USA, reductions in acute-care spending have driven more care to the outpatient setting. This may be one of the reasons for the observed increase in hospitals offering outpatient wound services as part of this cost shifting. For most hospitals, they have been productive cost centers as well as generating "spin-off” revenue for other hospital departments, such as imaging, the catheterization laboratory, and the operating room. However, the result has been a staggering increase in outpatient spending on "wound care services" estimated to be over 50 billion U.S. dollars per annum ${ }^{[2-6]}$.

Diagnosis-related groups (DRGs) effectively "capped” the cost of inpatient care by limiting reimbursement based on the number and severity of diagnoses. In contrast, the "fee for service" model of outpatient care has continued to reward both physicians and hospitals for performing high-cost, advanced therapeutics without a feedback mechanism for quality. Efforts are well underway to transition the U.S. outpatient payment system from one solely driven by volume to a model which in some way links cost to improved patient outcomes. Under the Affordable Care Act, the new Center for Medicare and Medicaid Innovation will direct a number of experimental programs in health care payment and delivery including Accountable Care Organizations (ACOs) ${ }^{[7]}$.

A detailed discussion of the design and mandate of ACOs is beyond our scope. However, briefly, ACOs are groups of providers that contract with CMS to take responsibility for patient care, agreeing to provide high-quality, patient-centered, evidenced-based care in the hope of reducing the costs incurred by those patients. In addition, under the Act, the recently launched Bundled Payments initiative will provide further incentive for health care providers who currently bill CMS separately for services supplied by hospitals, physicians, and other clinicians to bundle payments for a single "episode of care" ${ }^{[8]}$.Transitioning to value-driven payment that focuses on better health care at lower costs necessitates a reconsideration of physician financial incentives ${ }^{[9,10]}$. Many critics agree that to guarantee success, the ACO payment model needs to be implemented in gradual transition with partial fees-for-service/partial capitation. So, while an increasing number of both primary care and specialty physicians are salaried, even in the age of healthcare reform, physician payment remains largely volume driven, as noted in the recent commentary by Goroll and Schoenbaum ${ }^{[10]}$.

The trend toward employment of physicians is just beginning among hospital-based outpatient wound centers. Some of these agreements are part of actual Physician Hospital Organizations (PHOs), which are joint ventures between one or more hospitals and a group of physicians. The PHO plays an important role in the transition to integrated health care because of its focus on physician empowerment to make the ultimate decisions on patient care and have some say in negotiating pay ${ }^{[11]}$. It is acknowledged that in some instances (e.g. specialists and hospitals) preservation of fee for service or a mixed model of payment may make sense ${ }^{[10]}$. As hospitals and physicians negotiate the transition to new payment models, they need reliable revenue data upon which to base their decisions.

In most hospital-based outpatient wound care centers (WCC), transparency is lacking between the hospital and the physician with regard to revenue data. Even when practicing physically within the WCC, practitioners bill privately for their services and hospitals do not have not access to physician revenue information. On the other hand, wound center facility billing occurs within the hospital payment system so physicians do not have access to facility revenue data. Hospitals and physicians interested in developing a joint venture for wound care services need shared access to revenue data.

Although overall cost savings is a necessary aim, the ACO model is predicated on the concept that care will be quality driven. To accomplish this, ACOs rely on the adoption of electronic health records (EHRs) to facilitate quality measure reporting ${ }^{[3]}$ and to provide clinical decision support for evidence based care ${ }^{[11]}$. The recent "HITECH" Act (Health Information Technology for Economic and Clinical Health Act) ${ }^{[12]}$ set standards for the certification of such EHRs. However, the overarching goal of HITECH is to facilitate the nationwide electronic exchange of health information. Data exchange is accomplished by mandating the use of discrete data elements. Thus far, exchange of discrete data has been 
limited (e.g., vital signs). However, the bonus money available to physicians for EHR adoption requires them to meet certain "meaningful use" standards, and these become progressively more sophisticated over time. For example, by 2015, providers will have to incorporate 55\% of all laboratory tests as "structured data" within their EHR. In other words, the intent of the HITECH Act and its related incentive programs (including quality reporting initiatives), is to move clinicians away from the use of free text and dictation, toward the use of "structured language."

A few advanced EHRs have already achieved a high degree of structured-language usage. One wound-care-specific EHR makes almost exclusive use of pre-programmed, interoperable fields and as a result, is capable of internally and automatically performing the complex calculations required to automate billing functions. Thus, data on billed revenue has become an unanticipated benefit of the adoption of a particularly sophisticated electronic health record. This project utilized a sophisticated electronic medical record specifically designed to internally calculate the charges for both the physician and the facility in 3 hospital-based wound care centers. The goal was to determine the relative contribution of each entity (estimated revenue) to the total bill, and the portion attributable to procedures. These data may provide a starting point as providers begin the transition away from a purely volume-based delivery model

\section{Research methods}

\subsection{Data}

Outpatient wound center facility reimbursement for Medicare beneficiaries is defined by CMS in the Hospital Outpatient Prospective Payment System (HOPPS) ${ }^{[13]}$. We previously validated a method of calculating billing for the facility component using acuity scoring as measure of actual staff work performed. More than 200 elements of staff work contribute to the facility acuity scoring system ${ }^{[14]}$. The EHR internally calculates the facility level of service based on the documentation provided by hospital staff. Physician reimbursement of E/M services is determined by the 1995/97 Medicare Documentation Guidelines for physician documentation. The 1997 Documentation Guidelines for Evaluation and Management (E/M) Services contain 54 pages of detailed documentation requirements necessary to justify a specific level of service associated with different degrees of complexity of care. An outpatient visit may be billed at 1 of 5 levels of complexity. Complexity is reported by using 3 key components: (1) history (patient medical, family, and social history); (2) the physical examination; and (3) the physician's medical decision making. In order to bill any care during an initial visit, all 3 key components must be provided by the physician and documented in the patient's record. For subsequent visits, only 2 of the 3 key components have to be documented. Each key component contains 4 levels of difficulty. For initial visits, all 3 key components must support at least the minimum level of billing. Higher levels of billing require support from all 3 key components. Each of the 3 previously indicated sections (history, physical examination, and medical decision making), has 3 to 4 elements of documentation. For follow-ups, the level of service is determined by the most detailed documentation in 2 of the 3 categories. An initial visit must be supported by all 3 of the key components. With regard to medical decision making, points are possible for "cognitive effort” based on the number and complexity of active and chronic diagnoses (problems), number of labs interpreted, drugs prescribed, tests ordered, and so forth. Physician reimbursement of procedures is determined by the American Medical Association (AMA).

As discussed above, the complex nature of physician billing has encouraged the development of automated computer programs embedded within sophisticated EHRs to facilitate these calculations. While the 1997 CMS physician billing guidelines are stated only in narrative form, it is possible to construct a mathematical equation to calculate any level of service by taking those complex, detailed narrative descriptions, adding the number of fields needed to satisfy the requirements of each, and then adding the additional fields for activities, such as cognitive effort. According to the Healthcare Information and Management Systems Society ("HIMSS"), the ideal electronic health record would be one in which, "Physicians and clinical teams perform no extra tasks exclusively for medical record coding and reimbursement" ${ }^{[15,16]}$. With an internally automated system, clinicians do not later "decide" what level of service they have provided. The Intellicure $\mathrm{EHR}^{\mathrm{TM}}$ uses such a system to automatically calculate the level of service provided by 
physicians specific to the provision of wound care. Thus, the EHR runs an internal audit of both the facility and the physician documentation and calculates the appropriate level of service. Although the physician and the hospital facility level of service are both internally calculated by the EHR, the programming that determines these calculations are based on completely different rule sets as determined by their different Medicare coverage policies.

The Intellicure electronic health record (EHR) is certified by the ONC (Office of the National Coordinator) of Health Information Technology as a complete ambulatory care electronic health record in accordance with the certification criteria adopted by the Secretary of Health and Human Services (HHS). The "wound care-specific" physician billing module electronically audits the chart using a computer algorithm and then internally calculates the physician charges, including all procedural documentation for interventions, such as debridements or the application of bioengineered skin. Computers are present in every room allowing "point of service" documentation of all aspects of the patient's care. Data are maintained on servers hosted by the vendor, accessed through a secure portal. With the permission of the participating centers, EHR data are HIPAA de-identified and submitted to the U.S. Wound Registry (USWR). The USWR is a repository of linked de-identified data from the Intellicure EHRs. Through the Registry, de-identified, pooled data can be evaluated from all centers for the purpose of benchmarking reports and quality assurance. The registry is overseen by an independent institutional review board (IRB). However, such de-identified data do not contain protected health information (PHI) and are not subject to the privacy rule if all elements that could be used identify the individual have been removed.

\subsection{Data collection}

For this study, we analyzed billing data on each patient encounter for all patients seen at 3 busy wound care centers in the states of New York (NY), Texas (TX), and North Carolina (NC). From January 4 through December 31, 2010 (NC: July 1 through December 31), all patient encounters were evaluated, including the facility level of service and the physician-billed level of service, including procedures. We wished to have a standard "physician denominator unit” with which to compare hospital staff and so looked for clinics in which it would be easy to create a physician denominator unit of " 1 " in order to relate the hospital resources to that one doctor. Thus, we selected 3 facilities whose physician equivalent "FTE” (full time employee) number was equal to one.

\subsection{Cost estimation}

To estimate the anticipated revenue for the services, we utilized the Medicare-allowable rates adjusted for geographic practice cost indices for both the facility and the physician. These rates provide a good baseline estimate of revenue as more than $55 \%$ of patients in a wound care center are Medicare beneficiaries ${ }^{[17]}$. For the estimation of anticipated revenue for both the hospital and the physician, we analyzed all the procedures used in wound care which include hyperbaric oxygen therapy, debridements/surgical procedures, application of synthetic skin, the application of negative pressure wound devices, compression bandaging, offloading, vascular screening, and miscellaneous procedures/tests.

\section{Results}

A total of 6,762 patient visits occurred, comprising 887 initial consultations and 5,875 follow-up visits. At both NY and TX wound care centers there were 2 physicians who worked part time as 1 FTE (full time equivalent) while in the NC wound care center there were 7 physicians who worked part time as 1 FTE. The patient to physician ratios based on FTEs were 454 (NY), 1104 (TX), and 442 (NC).

More than 55\% of patients were Medicare beneficiaries. We estimated all reimbursement (for both the hospital and the physician) based on Medicare allowables for each service as Medicare is typically the lowest of the various payers. Thus, our estimated reimbursement is a conservative one for both entities. The average anticipated revenue per physician, based upon Medicare reimbursement was calculated as $\$ 449,708$ while the average anticipated revenue for the facility, based on 
one physician's activities was $\$ 1,469,461$ (Table 1). Physician revenues constituted almost a quarter of total revenue while the range (lowest to highest) of the proportion varied between 21 and 29\%. In terms of anticipated global revenue, procedures provided almost three quarters of the revenue on average although the range between the 3 clinics was $66-82 \%$ (Table 2).

Table 1. Facility and physician revenue for $\mathrm{E} / \mathrm{M}$ and procedure revenues by clinic

\begin{tabular}{|c|c|c|c|c|c|c|}
\hline \multirow{2}{*}{ Clinic } & \multicolumn{2}{|c|}{ E/M Revenue (\$) } & \multicolumn{2}{|c|}{ Procedure Revenue (\$) } & \multicolumn{2}{|l|}{ Totals (\$) } \\
\hline & Physician & Facility & Physician & Facility & Physician & Facility \\
\hline Texas & 148,984 & 178,290 & 133,549 & 500,078 & 282,533 & 678,369 \\
\hline New York & 196,779 & 215,189 & 289,144 & $1,568,045$ & 485,923 & $1,783,234$ \\
\hline North Carolina & 348,029 & 408,801 & 232,639 & $1,537,980$ & 580,668 & $1,946,781$ \\
\hline TOTAL & 693,792 & 802,281 & 655,332 & $3,606,104$ & $1,349,125$ & $4,408,384$ \\
\hline
\end{tabular}

Table 2. Global revenue by E/M and procedure, and percentage of revenue related to procedures

\begin{tabular}{lllll}
\hline Clinic & $\begin{array}{l}\text { Global Revenue } \\
\text { E/M (\$) }\end{array}$ & $\begin{array}{l}\text { Global Revenue } \\
\text { Procedure (\$) }\end{array}$ & $\begin{array}{l}\text { Total Global } \\
\text { Revenue (\$) }\end{array}$ & $\begin{array}{l}\text { Revenue Related } \\
\text { to Procedures (\%) }\end{array}$ \\
\hline Texas & 327,274 & 633,627 & 960,902 & 66 \\
New York & 411,968 & $1,857,189$ & $2,269,157$ & 82 \\
North Carolina & 756,830 & $1,770,619$ & $2,527,450$ & 70 \\
TOTAL & $1,496,073$ & $4,261,436$ & $5,757,509$ & 74 \\
\hline
\end{tabular}

Table 3. Percentage of revenue for procedures by clinic location and facility/physician

\begin{tabular}{|c|c|c|c|c|c|c|}
\hline \multirow{2}{*}{ Procedure } & \multicolumn{3}{|c|}{ Facility Revenue (\%) } & \multicolumn{3}{|c|}{ Physician Revenue (\%) } \\
\hline & TX & NY & NC & TX & NY & NC \\
\hline Compression bandaging & 0.1 & 0.5 & 0.0 & 0.2 & 1.3 & 0.0 \\
\hline Debridement and surgical procedures & 80.1 & 9.7 & 24.4 & 67.1 & 11.3 & 44.9 \\
\hline Hyperbaric oxygen therapy & 0.0 & 77.0 & 72.4 & 0.0 & 29.7 & 34.2 \\
\hline Miscellaneous procedures/tests & 0.2 & 2.6 & 1.0 & 0.3 & 4.3 & 1.3 \\
\hline Negative pressure dressing placement & 0.3 & 0.7 & 0.1 & 0.4 & 1.2 & 0.3 \\
\hline Offloading & 2.8 & 0.3 & 0.1 & 3.5 & 0.5 & 0.2 \\
\hline Synthetic skin placement & 16.4 & 6.1 & 1.7 & 27.8 & 24.7 & 16.8 \\
\hline Vascular screening & 0.1 & 3.2 & 0.2 & 0.6 & 26.9 & 2.3 \\
\hline
\end{tabular}

The percentage of facility revenues by procedures was dominated by debridement and surgical procedures in one clinic that was not involved in hyperbaric oxygen therapy (HBOT) at $80.1 \%$, but in the 2 other clinics that were involved in HBOT, this procedure dominated (77.0 and 72.4\%) (Table 3). In the latter 2 clinics, debridement and surgical procedures constituted the second largest category (9.7\% and $24.4 \%)$ while in the first clinic synthetic skin replacement was the second highest category. Physician revenue was more diverse by procedure: the 2 top revenue-gathering procedures were debridement/surgical procedures (67.1\%) and synthetic skin placement (27.8\%) in the Texas clinic, while they were HBOT and vascular screening in the New York clinic (29.7 and 26.9\%, respectively), and debridement/surgical procedures and HBOT in the North Carolina clinic (44.9 and 34.2\%, respectively) (Table 3).

\section{Discussion}

If the ACO provides most of the primary care for a patient, it can receive incentive payments from Medicare. In order to receive incentive payments, an ACO must report quality measures, exceed certain thresholds in quality and patient experience, and generate a savings over baseline costs as established by Medicare. If the ACO meets all quality and cost 
goals set by CMS, it can receive a substantial portion of the savings. If it fails to reduce costs, it would share in the increased expense by paying Medicare back the incentive money it has received. The hope is that this structure will contain the runaway cost of outpatient "fee for service" medicine while rewarding efficient patient care. However, according to the American Hospital Association the percentage of hospitals with PHOs has steadily declined from 27\% in 1999 to $18 \%$ in $2008{ }^{[18]}$. Although we do not know the precise reason for this decline, in order for an ACO or a PHO to be successful, it must have a sophisticated EHR system capable of calculating and tracking both financial and performance (quality) measures. Consequently, we felt it important to demonstrate the ways a sophisticated EHR can provide financial data as hospitals evaluate opportunities for physician and facility business arrangements.

Of the total combined revenue generated by both entities, E/M charges represent only $26 \%$. Procedural revenue represents on average, $74 \%$ of the revenue generated by both the physician and the facility in the WCC. Procedures include hyperbaric oxygen therapy, debridement, and application of bioengineered skin. However, with regard to total procedural revenue, the physician's portion represents only $15 \%$, whereas the hospital portion is $85 \%$ of the total amount billed. This is because Medicare has created a billing structure acknowledging the high overhead costs borne by the facility, including staffing and equipment, which are far greater than those borne by the physician whose overhead in this scenario consists of malpractice insurance, benefits, and billing services. This is reflected by the numbers in Table 1 . The percentage of total revenue coming from procedures is dependent on the type of procedures carried out at the WCC. In the case of the New York clinic in which revenue is primarily from HBOT and synthetic skin placement, the overall percentage of total revenue related to procedures is $82 \%$. Contrast this to the Texas clinic in which there are no HBOT procedures carried out. The most common procedures there are synthetic skin placement and debridement with the total revenue from procedures estimated at $66 \%$. Thus we see that some procedures are more "profitable" than others, although they may also be associated with higher overhead.

The current system encourages the performance of more highly remunerative procedures as a method of revenue generation, regardless of their benefit to the patient. Transitioning to some other reimbursement model will be difficult when powerful incentives exist to keep the system the same, and some "baseline" amount of revenue is necessary for both the hospital and the physician if they are to provide any services. Thus, if a managed system limits procedural revenue, how much "incentive money" would be required to compensate for these losses in order for hospitals and physicians to continue to operate? How can the physician and the hospital share the remaining revenue and be equally incentivized in the provision of quality care which is not based on maximizing interventions? Our data at least provide some benchmark of the "current state" as administrators grapple with the economics of the transition and current financial expectations.

The importance of internal automation within an EHR for both facility and physician billing cannot be overstated from the standpoint of compliance. With "checklist" systems, it is necessary to have a human review the chart (even an electronic chart that uses text fields for data entry) to ensure that the documentation is actually present. However, within an EHR making extensive use of structured language, points only accrue if documentation is actually present. Some have suggested that this method may result in physicians billing for higher levels of service than they deserve. However, the burdensome nature of hand-written charting means that clinicians often fail to document all they actually have done, and thus, do not bill for all they might, rather than the reverse ${ }^{[19]}$. More importantly, as physicians are driven toward "point of care" charting, the decision support and performance measures needed for successful PHOs become integrated within the health information system. The hope is that this will further improve compliance with evidence based care. Given the specific requirements placed upon ACOs for quality measures reporting, implementation of decision support, and electronic prescribing, it will be impossible to create them without a sophisticated EHR ${ }^{[20]}$.

This study has a number of limitations. First, because it was a pilot study, only data at 3 clinics were examined. Second, while we do not believe that the method by which we created a 1-FTE equivalent at each clinic severely distorted any data, it could have introduced some bias indirectly through population selection, including the patient-physician ratio, Medicare:private provider ratio, or overall wound severity or number/severity of patient comorbidities. 


\section{Conclusions}

The analysis of parties' relative contributions to the global fee based on their separate responsibilities in wound care outpatient settings was considerably different. On average three quarters of WCC revenue is procedural in nature compared to evaluation and management. Thus, we have an uncontrolled system in which ever-increasing numbers of procedures can occur without regard to patient-centered outcomes. If procedural revenue is curtailed, incentives for providing cost-efficient care must be sufficient to motivate both hospitals and doctors to support the new model. The next step in will be to determine the relative value of interventions provided to WCC patients in order to determine where savings can be achieved. A sophisticated EHR designed specifically for the purpose of capturing charge data, provides the mechanism for future cost effectiveness studies.

\section{Competing interests}

CEF, VW, DW, and TT are employees of Intellicure, Inc. MJC is a paid consultant to Intellicure, Inc.

\section{Author contributions}

CEF conceived the study and drafted the manuscript with help from MJC. VW, BT, and DW collected the data; VW, MJC, and DW analyzed the data. All authors revised the manuscript critically for important intellectual content.

\section{References}

[1] Centers for Medicare and Medicaid Services: Medicare Coverage Advisory Committee [Internet]. Available from: http://www.cms.hhs.gov/FACA/downloads/id28a.pdf?origin=globalsearch\&page=/mcd/viewmcac.asp\&mid=28\&where=index

[2] Fife CE, Carter MJ, Walker D, Thomson B: Wound care outcomes and associated cost among patients treated in US outpatient wound centers: Data from the US Wound Registry. Wounds. 2012; 24: 10-17.

[3] Kuhn BA, Coulter SJ: Balancing ulcer cost and quality equation. Nurs Econ. 1992; 10: 353-359.

[4] Hess CT: Putting the squeeze on venous ulcers. Nursing. 2004; 34(Suppl Travel): 8-13.

[5] Driver VR, Fabbi M, Lavery LA, Gibbons G: The costs of diabetic foot: The economic cases for the limb salvage team. J Am Podiatr Med Assoc. 2010; 100: 335-341. PMid:20847346

[6] Gordon MD, Gottschlich MM, Helvig EI, Marvin JA, Richard RL: Review of evidence-based practice for the prevention of pressure sores in burn patients. J Burn Care Rehabil. 2004; 25: 388-410. PMid:15353931 http://dx.doi.org/10.1097/01.BCR.0000138289.83335.F4

[7] Goldsmith J: Accountable care organizations: The case for flexible partnerships between health plans and providers. Health Aff (Millwood). 2011; 30: 132-140. PMid:21209435 http://dx.doi.org/10.1377/hlthaff.2010.0782

[8] Sood N, Huckfeldt PJ, Escarce JJ, Grabowski DC, Newhouse JP: Medicare's bundled payment pilot for acute and postacute care: Analysis and recommendations on where to begin. Health Aff (Millwood). 2011; 30: 1708-1717. PMid:21900662 http://dx.doi.org/10.1377/hlthaff.2010.0394

[9] Burke T: Accountable Care Organizations. Public Health Reports. 2011; 126: 875-78. PMid:22043105

[10] Goroll AH, Schoenbaum SC: Payment reform for primary care within the accountable care organization. A critical issue for health system reform. JAMA. 2012; 308: 577-578. PMid:22871868 http://dx.doi.org/10.1001/jama.2012.8696

[11] James MH: Navigating the road ahead. Lessons from a pioneer ACO. Healthc Financ Manage. 2012; 66: 64-9. PMid:22931028

[12] Arlotto P: 7 strategies for improving HITECH readiness. Healthc Financ Manage. 2010; 64: 90-4, 96. PMid:21061823

[13] Department of Health and Human Services: Office of Inspector General; Medicare Program; Prospective Payment System for Hospital Outpatient Services. Federal Register. 2000; 65: 18450-18451.

[14] Fife CE, Walker D, Farrow W, Otto G: Wound center facility billing: A retrospective analysis of time, wound size, and acuity scoring for determining facility level of service. Ostomy Wound Manage. 2007; 53: 34-44. PMid:17264354

[15] Handler T, Holtmeier R, Metzger J, Overhage M, Taylor S, Underwood C: HIMSS Electronic Health Record Definitional Model Version 1.1 [Internet]. Available from: http://www.himss.org/content/files/ehrattributes070703.pdf

[16] Fife CE, Wall V, Carter MJ, Walker D, Thomson B: Examining the relationship between physician and facility level-of-service coding in outpatient wound centers: Results of a multicenter study. Ostomy Wound Manage. 2012; 58: 20-28. PMid:22391954

[17] Fife CE, Carter MJ, Walker D, Thomson B: A retrospective data analysis of antimicrobial dressing usage in 3,084 patients. Ostomy Wound Manage. 2010; 56: 28-42. PMid:20368672 
[18] American Hospital Organization: Trendwatch Chartbook 2011 [Internet]. Available from: http://www.aha.org/research/reports/tw/chartbook/2010/appendix2.pdf

[19] King MS, Sharp L, Lipsky MS: Accuracy of CPT evaluation and management coding by family physicians. J Am Board Fam Pract. 2001; 14: 184-192. PMid:11355050

[20] Singer S, Shortell SM: Implementing accountable care organizations. JAMA. 2011; 306: 758-759. PMid:21828308 http://dx.doi.org/10.1001/jama.2011.1180 